\title{
Plaque-Type Psoriasis Treated with Calcipotriene Plus Betamethasone Dipropionate Aerosol Foam: A Prospective Study on Clinical and Dermoscopic Predictor Factors in Response Achievement and Retention
}

Enzo Errichetti · Margherita Croatto $\cdot$ Luca Arnoldo $\cdot$ Giuseppe Stinco

Received: May 11, 2020 / Published online: June 12, 2020

(C) The Author(s) 2020

\section{ABSTRACT}

Introduction: Little information on possible local factors that can influence the achievement and retention of response of plaque-type psoriasis to calcipotriene plus betamethasone dipropionate aerosol foam is available. The aim of this study was to assess possible correlations between baseline clinical/dermoscopic features of psoriatic plaques and therapeutic response, and between residual dermoscopic findings in clinically improved/healed lesions and posttreatment relapse.

Methods: For each target lesion, we calculated the local psoriasis severity index and assessed dermoscopic findings at baseline and at the end of a 4-week treatment, correlating the therapeutic outcome with the initial clinical and dermoscopic features. The lesions were also followed for a 4-week post-treatment period, and possible associations between relapse and (1) baseline clinical/dermoscopic features and

Digital Features To view digital features for this article go to https://doi.org/10.6084/m9.figshare.12383255.

E. Errichetti $(\bowtie) \cdot$ M. Croatto · G. Stinco Department of Medical Area, Institute of Dermatology, University of Udine, Udine, UD, Italy e-mail: enzoerri@yahoo.it

L. Arnoldo

Department of Medical Area, Institute of Accreditation and Quality, University of Udine, Udine, UD, Italy
(2) dermoscopic findings detected at the end of the treatment were assessed.

Results: A total of 105 lesions from 35 patients were included in the analysis. After 4 weeks of therapy, 13 lesions showed no/limited improvement, while partial and optimal response were observed in 51 and 41 plaques, respectively. Poor outcomes were correlated with both legs localization and degree of lesion infiltration at baseline. Similarly, presence of globular vessels at baseline dermoscopy was more commonly associated with no/limited response and lesions on the legs, particularly those showing resistance to treatment. A correlation was also found between dotted vessels on the baseline dermoscopic examination and good outcomes (partial/optimal response). After a 4 -week post-treatment follow-up, $58.7 \%$ of the lesions achieving improvement at the end of the therapy showed relapse, with a correlation between recurrence and vessel persistence on dermoscopy at the end of the therapy.

Conclusion: Clinical response of plaque-type psoriasis to calcipotriol/betamethasone dipropionate spray foam may be adversely affected by the degree of infiltration of lesions at baseline and by legs localization, and the presence of globular vessels at the baseline dermoscopic assessment is related to poor outcomes. A significant post-treatment relapse rate was observed, and persistence of vascular changes on dermoscopy seems to play a role in promoting disease recurrence. 
Keywords: Dermoscopy; Monitoroscopy; Predictor factors; Psoriasis; Relapse; Therapy

\section{Key Summary Points}

Why carry out this study?

Little information on possible local factors influencing response achievement and retention of plaque-type psoriasis to a 4-week course of treatment with calcipotriene plus betamethasone dipropionate aerosol foam is available.

Correlations between baseline clinical/dermoscopic features of psoriatic plaques and therapeutic response as well as between residual dermoscopic findings in clinically improved/healed lesions at the end of treatment and post-treatment relapse were assessed.

\section{What was learned from the study?}

Poor therapeutic outcomes (no/limited response) were associated with lesions located on the legs, degree of baseline lesion infiltration and globular vessels on baseline dermoscopy, while dotted vessels on baseline dermoscopic examination were correlated with good outcomes (partial/optimal response).

After a 4-week post-treatment follow-up, more than half of the lesions achieving improvement at the end of the therapy showed relapse, with a correlation between recurrence and persistence of vessels on dermoscopy at the end of the treatment.

\section{INTRODUCTION}

Calcipotriene plus betamethasone dipropionate aerosol foam is an innovative topical treatment for plaque-type psoriasis which has been shown to be well-tolerated and significantly effective in terms of both objective improvement and itching/pain relief [1-4], with consequent relevant amelioration of patients' quality of life, which is often compromised in this condition [5].

Whereas it has been demonstrated that the efficacy of such a treatment is independent of the patient's body mass index and disease extent/severity $[1,2]$, there is a lack of data regarding possible local (lesional) factors influencing therapeutic outcomes. In this regard, we recently showed that the therapeutic response of psoriatic patients to narrowband ultraviolet $B$ (NB-UVB) phototherapy may be significantly influenced by the clinical features of each lesion, with plaques localized on the legs and/or showing more scaling/infiltration being associated to treatment resistance [6]. Additionally, in the same study, we also demonstrated that dermoscopic assessment may highlight subclinical response predictors, with dotted and globular vessels related to good and bad outcomes, respectively [5]. Similarly, Lallas et al. observed that the presence of purpuric dots on dermoscopy may be an early positive predictor of response in psoriatic patients treated with biologic agents [7].

In addition to the possible role of dermoscopy to predict therapeutic response according to baseline features, such a technique might also have a potential role in assessing the likelihood of disease recurrence. Indeed, some lesions achieving complete clinical healing after treatment may still display residual subclinical abnormalities visible on dermoscopic examination (i.e. dotted/globular vessels) which might facilitate disease recurrence [8].

In this prospective study, our aim was to assess possible correlations between baseline clinical/dermoscopic features of psoriatic plaques and therapeutic response, as well as between residual dermoscopic findings in clinically improved/healed lesions and post-treatment relapse, in order to better optimize the use of calcipotriene and betamethasone aerosol foam. 


\section{METHODS}

Adult patients (aged $\geq 18$ years) suffering from moderate-to-severe plaque-type psoriasis (Psoriasis Area and Severity Index [PASI] score $>10$ ) for at least 6 months scheduled to undergo a 4-week (once-daily) treatment with calcipotriene plus betamethasone dipropionate aerosol foam were selected for this study (enrolment started in March 2019 and ended in November 2019). Patients currently taking antipsoriatic treatments or those who had used systemic/topical anti-psoriatic drugs in the prior 12 weeks as well as subjects who had been exposed to sun during the previous 4 weeks were not considered to be eligible. We also excluded patients suffering from other relevant dermatoses, calcium metabolism disorders and severe kidney failure or liver dysfunction as well as pregnant or breastfeeding women.

Three target psoriatic plaques were randomly selected from each participant to be documented and followed. Lesions located on the scalp, face, folds and palmoplantar areas were not considered in the analysis. Anatomical localization of each plaque was identified by taking photographs and (when possible) recording distances from static benchmarks (e.g. bony prominences). Evaluations (both clinical and dermoscopic) were performed by two experienced researchers in consensus; in case of disagreement a third researcher was involved.

The study protocol involved three visits by the patients to the clinic, which is in line with our routine clinical practice in the management of patients with moderate-to-severe psoriasis undergoing calcipotriene plus betamethasone aerosol foam therapy: a baseline visit (T0), assessment at the end of therapy (week 4 [T4]) and a follow-up evaluation after 8 weeks from baseline (T8). Notably, between T4 and T8, patients were asked not to apply anything on the treated areas, with the exception of emollients. Patients requiring additional systemic/topical anti-psoriatic treatments during the whole study period were excluded from the analysis.
At the baseline (T0), each selected lesion was evaluated from a clinical and dermoscopic aspect. In the clinical evaluation, we calculated the Local Psoriasis Severity Index (LPSI), which corresponds to the sum of erythema, scaling/ desquamation and induration/infiltration severity on a 5 -point scale (0-4), with a total score ranging from 0 to 12 . The dermoscopic analysis consisted of assessing the presence of several parameters that have been established in the literature as standardized criteria for the assessment of non-neoplastic dermatoses [6, 9]; these include dotted vessels (uniform, clustered, peripheral, reticular or unspecific distribution), globular vessels (uniform, clustered, peripheral, reticular or unspecific distribution), non-dotted/non-globular vessels (uniform, clustered, peripheral, reticular or unspecific distribution), white scales (diffusely distributed, patchily distributed, central or peripheral) and purple (haemorrhagic), white and brown areas (dots, globules, lines or structureless areas). Dermoscopic assessment was performed using a polarized hand-held device $(10 \times$ magnification), with attention on minimizing the pressure in order to preserve vessel morphology and enhance visualization.

After 4 weeks of therapy (T4), we evaluated clinical improvement as limited response (LPSI improvement $<50 \%$ ), partial response (LPSI improvement of $50-75 \%$ ) or optimal response (LPSI improvement $>75 \%$ ) and correlated the therapeutic outcome with initial clinical and dermoscopic features. We also performed a dermoscopic examination to assess subclinical changes of the lesions.

Finally, at week 8 (T8), we evaluated the change in the LPSI between week 4 and week 8 ( $\triangle$ LPSI post-treatment) to assess the rate of posttreatment relapse (defined as the loss of at least $50 \%$ of LPSI amelioration reached at the end of treatment) in lesions achieving improvement (at least 50\% compared to baseline) at $\mathrm{T} 4$. Additionally, we evaluated possible associations between relapse and (1) baseline clinical/dermoscopic features, (2) lesion localization and (3) dermoscopic findings at the end of the treatment (week 4). 


\section{Statistical Analysis}

All analyses were performed using the SPSS version 20 software (IBM Corp., Armonk, NY, USA). Data are expressed as the mean \pm standard deviation (SD) or as percentages. To assess the normality of the distribution we performed the Chi-square and Shapiro-Wilk tests; the Kruskal-Wallis and Mann-Whitney tests were used as non-parametric tests. All tests were two tailed, and $p$ value of $<0.05$ was deemed to be statistically significant.

\section{Compliance with Ethics Guidelines}

The study was conducted in accordance with the Helsinki Declaration of 1964 and its later amendments and all the participants gave their informed consent to take part in the study and use personal records/photos for scientific purpose. Local ethical committee approval was obtained from the Comitato Etico Unico Regionale (C.E.U.R.) del Friuli Venezia Giulia (CEUR-2018-OS-140-ASUIUD).

\section{RESULTS}

A total of 105 target lesions from 35 patients (16 females and 19 males; average age [ \pm SD] $56 \pm 16$ years; average lesion duration 10 weeks) were included in the analysis. Most lesions were located on the trunk (43/105; $41 \%)$, legs $(20 / 105 ; 19.1 \%)$ and extensor surfaces of the knees and elbows $(18 / 105 ; 17.1 \%)$, whereas thighs and upper arms were involved in $7.6 \%(8 / 105)$ and $15.2 \%(16 / 105)$ of cases, respectively. Mean LPSI at baseline was $7.84 \pm 1.3$, with a mean score for erythema, scaling/desquamation and induration/infiltration of $2.6 \pm 0.7$ (for all such variables).

After 4 weeks of therapy, a total of 13 (12.4\%) target lesions did not improve or reached only a limited response, while partial and optimal response were observed in 51 $(48.6 \%)$ and $41(39.0 \%)$ target lesions, respectively. A total of 32 lesions achieved a LPSI score of 0 or $1(30.5 \%)$.
Notably, there was a significant correlation between anatomical localization and clinical response, with resistant (no or limited response) lesions more commonly located on the legs and extensor areas. In more detail, plaques located on the legs showed a 13-fold higher probability to show no/limited response than lesions located on the trunk or upper arms (odds ration [OR] 13.2, 95\% confidence interval [CI] 2.8-63.8). No significant association was observed between clinical response and either baseline LPSI or baseline erythema/scaling, while a higher baseline infiltration score correlated with a poor therapeutic outcome (OR 3.9, 95\% CI 1.3-11.9). Finally, no association was observed between clinical response and either lesion duration or gender. Analysis of the dermoscopic findings (Table 1) revealed a significant correlation between the presence of globular vessels at baseline and no/limited response (OR 9, 95\% CI 3.5-12.9) (Fig. 1). Interestingly, such vessels were more frequently observed in lesions on the legs than in those on other anatomical regions, while dotted vessels were more frequently present in lesions on the trunk $(p<0.05)$. Moreover, considering only psoriatic plaques located on the legs, globular vessels were still significantly more common in non-responding lesions than in lesions achieving improvement $(p<0.05)$. Finally, dotted vessels were associated with good outcomes (partial/optimal response) (OR 5.7, 95\% CI 1.5-22.1) (Fig. 2).

After a 4-week post-treatment follow-up, $58.7 \%(54 / 92)$ of the lesions showed relapse. A similar (no statistical difference) relapse rate was observed when only lesions reaching complete/ nearly complete clinical improvement (LPSI 0 or 1$)$ at T4 were considered $(50 \% ; 16 / 32)$. No correlation between response retention and either lesion localization or baseline LPSI was observed. Similarly, response retention was not affected by baseline erythema, scaling/desquamation, induration/infiltration or baseline dermoscopic features. On the other hand, a statistically significant association was found between relapse and persistence of vessels at the end of the therapy when considering both all the lesions regardless of degree of improvement at T4 (OR 17.3 CI 95\% 6.0-49.7) and only 
Dermatol Ther (Heidelb) (2020) 10:757-767

761

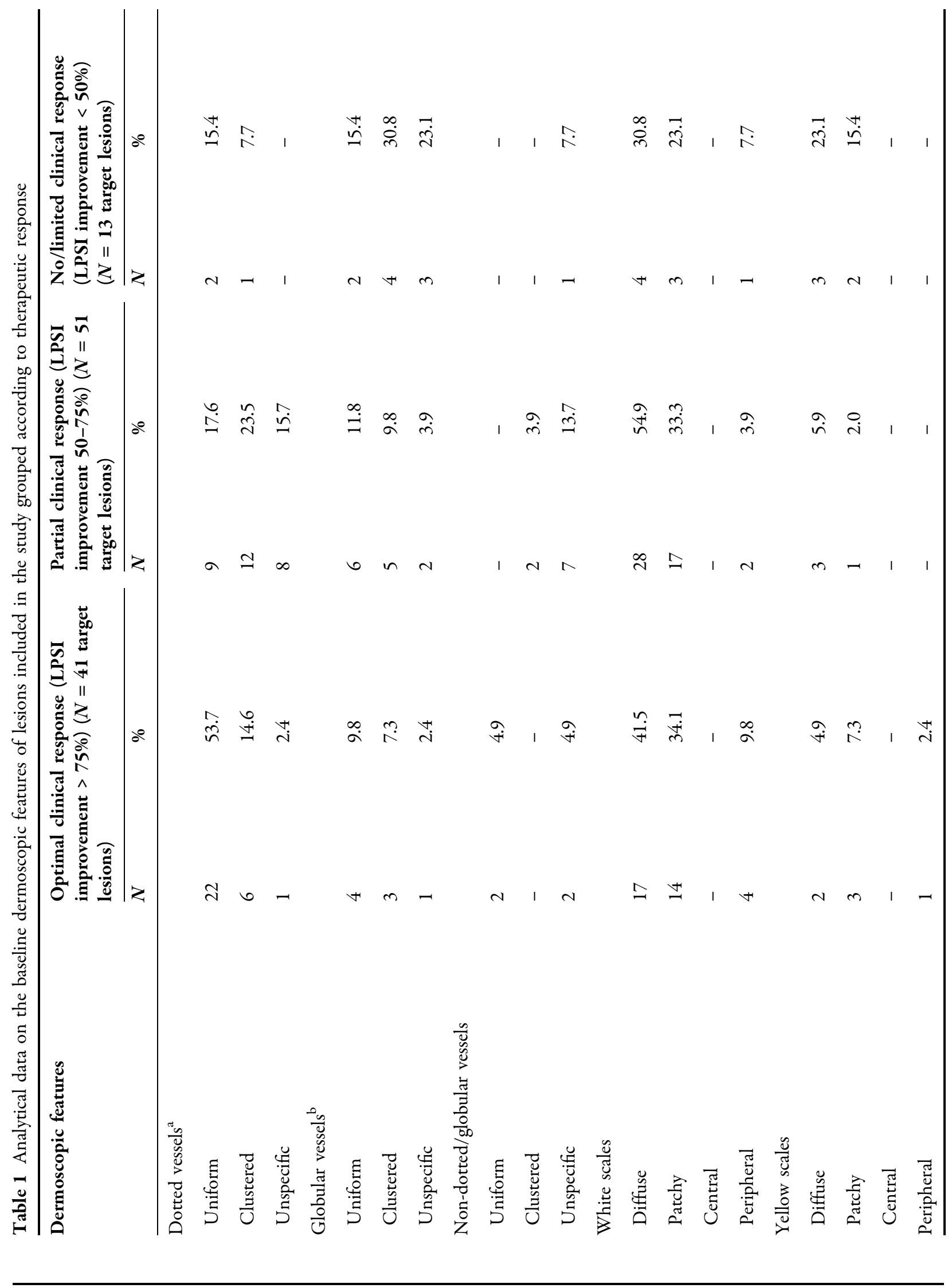

么 Adis 


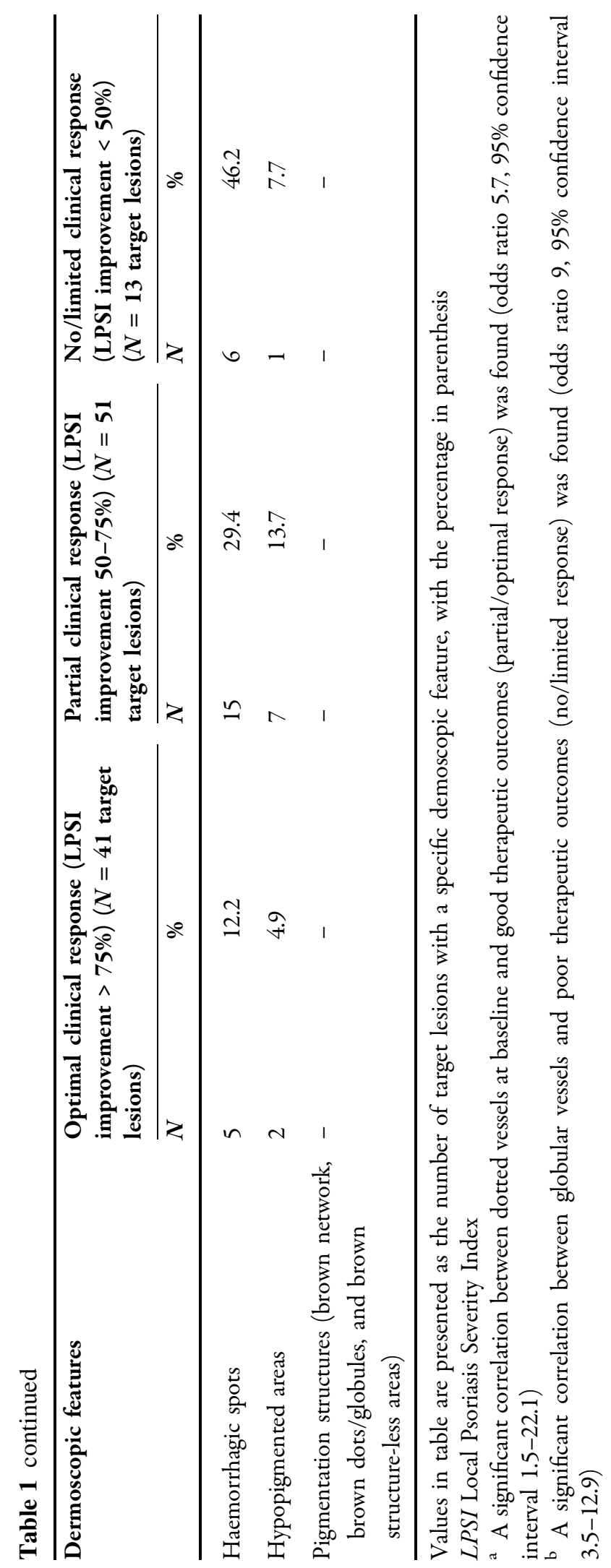




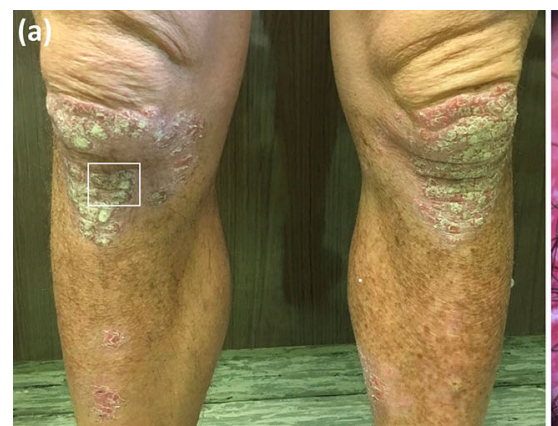

Fig. 1 a, b Clinical picture of a target psoriatic plaque on the right leg (white square) at baseline (a), with corresponding dermoscopy image showing presence of uniform globular vessels (better visible in inset) and diffuse
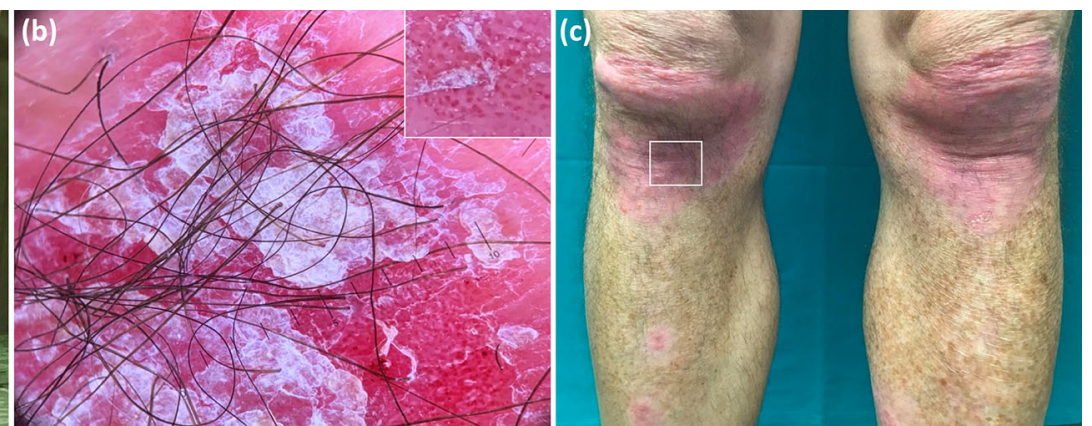

white scaling $(\mathbf{b})$. c Clinical aspect of the same lesion after 4 weeks of therapy with calcipotriene plus betamethasone dipropionate aerosol foam showing limited improvement (Local Psoriasis Severity Index improvement of $<50 \%$ )
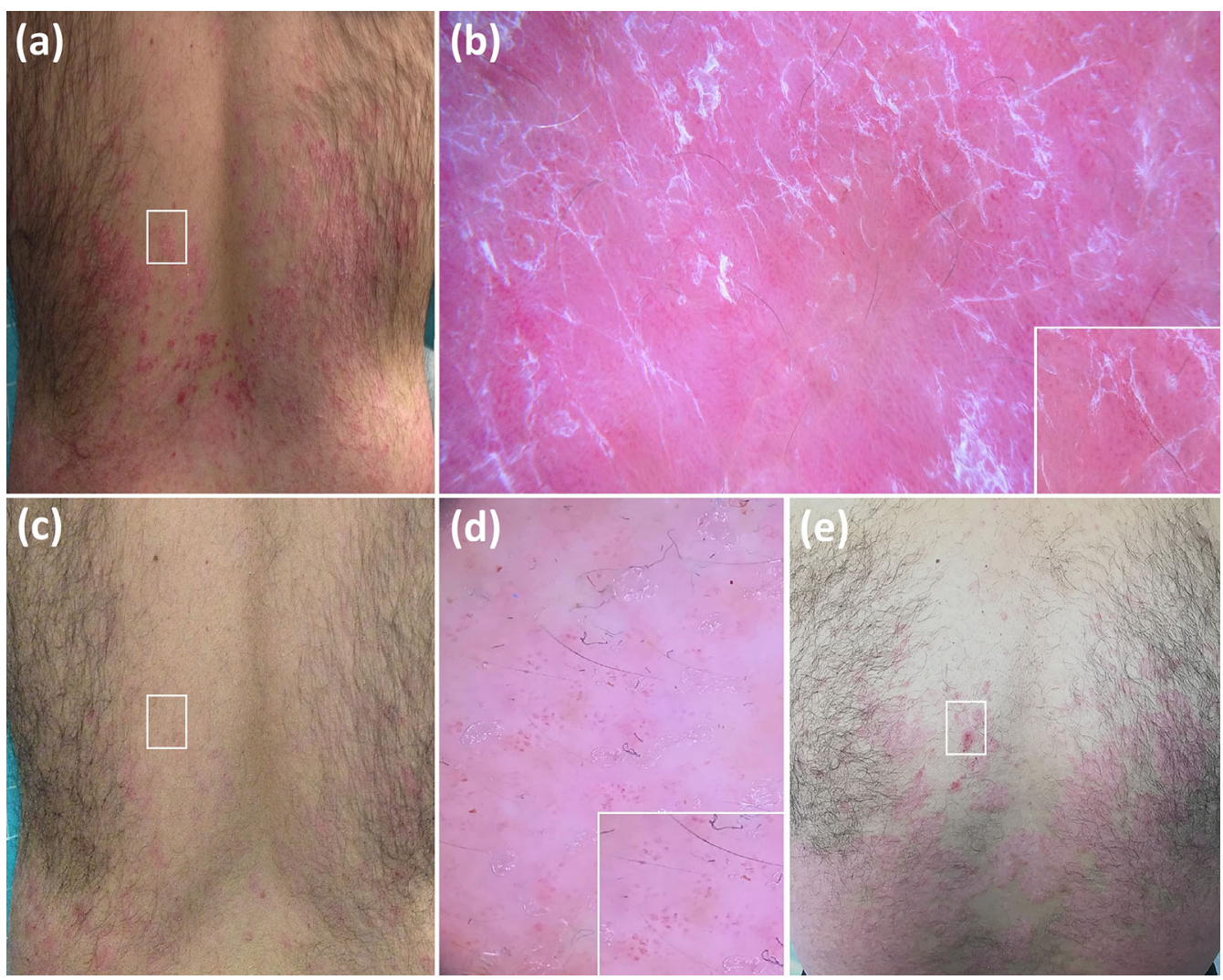

Fig. 2 a, b Clinical picture of a target psoriasis plaque (white square) at baseline (a), with corresponding dermoscopy image showing presence of uniform dotted vessels (better visible in inset) (b). c, d After 4 weeks of therapy with calcipotriene plus betamethasone dipropionate

aerosol foam the target lesion (white square) showed complete clinical healing (c), but vessels were still evident on dermoscopy (better visible in inset) (d). e Clinical recurrence of the lesion (white square) after 4 weeks of post-treatment follow-up (T8) 


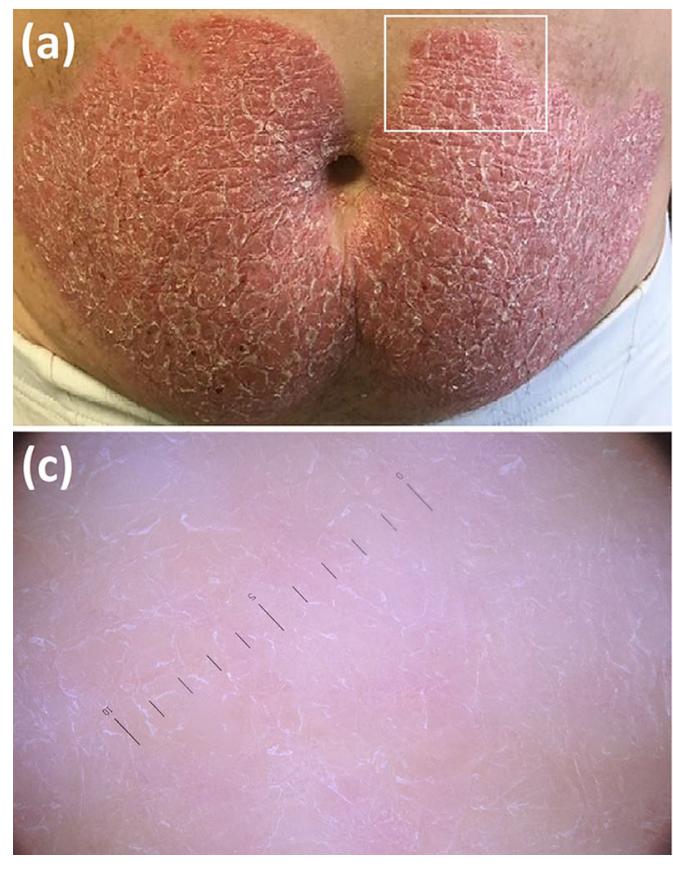

Fig. 3 a-c Clinical pictures of a target psoriatic plaque on the abdomen (white square) at baseline (a) and after 4 weeks of treatment with calcipotriene plus betamethasone dipropionate aerosol foam with complete healing

lesions reaching a complete/nearly complete clinical healing (LPSI 0 or 1) at T4 (OR 21.0 CI95\% 3.3-135.5) (Figs. 2, 3).

\section{DISCUSSION}

Most of the studies published to date that have evaluated the efficacy of calcipotriene plus betamethasone dipropionate aerosol foam for the treatment of plaque-type psoriasis have focused on general disease improvement without any assessment of local (lesional) outcomes. The consequence has been a lack of data on the effects of this therapy on specific target lesions and possible local factors that may influence therapeutic response. Only one prospective study has specifically evaluated the efficacy of calcipotriene plus betamethasone dipropionate aerosol foam on target lesions, with the results showing a significant improvement of local outcomes in the analysed plaques [10]. Our study further confirms the "local" efficacy of this therapy, although we also observed a small

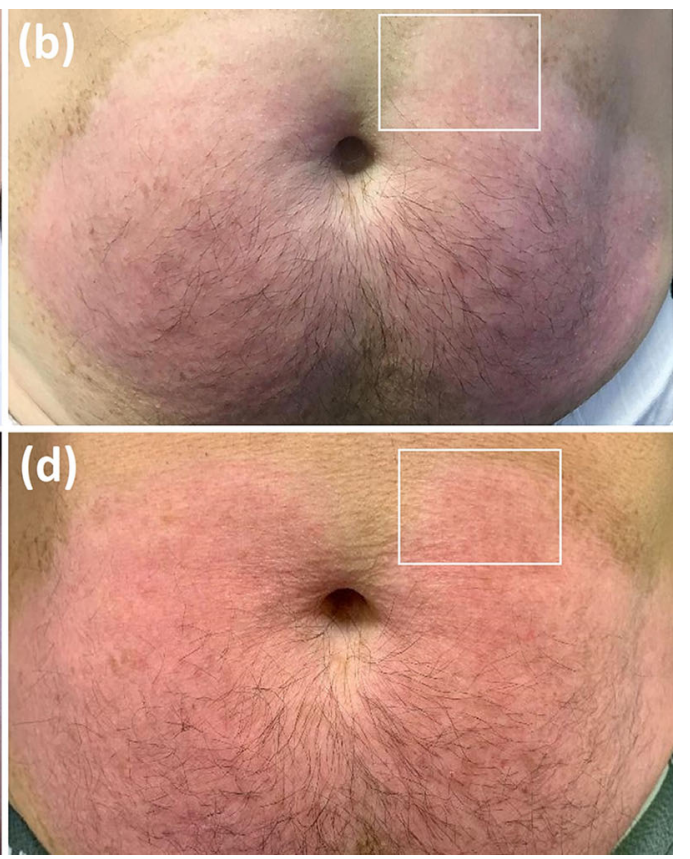

(white square) based on both clinical (b) and dermoscopy (absence of vessels) (c) findings. d No recurrence was evident after 4 weeks of post-treatment follow-up

subgroup (12.4\%) of lesions that either did not improve or achieved only a limited response. One possible explanation for this difference may be that only plaques on the elbows and knees were included in the previous study [10]. Indeed, our analysis showed that resistant lesions were mainly located on the legs, thereby supporting the hypothesis that anatomical localization may affect clinical response. On the other hand, we did not find a correlation between therapeutic response and other clinical variables, apart from the infiltration degree of the lesions, with thicker plaques showing poor outcomes.

The negative impact of the localizion of lesions on legs and high lesion infiltration degree on therapeutic efficacy is consistent with the results from Errichetti and Stinco's (two of the authors of the present invesigation) study on psoriatic patients treated with NB-UVB [6]. However, differently from our previous study, we did not observe any association between lesion scaling degree and poor response to calcipotriene plus betamethasone dipropionate 
aerosol foam, likely due the high penetration of the treatment through the corneum layer [11].

While the association between lesion infiltration and reduced response is easily explained by the physical barrier of the plaque to drug penetration, the actual mechanisms underlying the correlation between the lesion localization on legs and poor therapeutic outcomes is yet to be completely understood, although vascular factors has been speculated to be involved [6]. Indeed, as observed in the present study and our previous analysis [6], psoriatic plaques localized on the legs more commonly feature vessels of a larger size on dermoscopy (i.e. globular vessels), particularly those lesions showing limited/no clinical response, thereby indicating a possible association between vascular dilation/increased vascular flow and therapeutic resistance in this anatomical region [6]. This speculation is further supported by the different response rate to calcipotriene plus betamethasone dipropionate aerosol foam according to vessel size observed on dermoscopy, with the presence of globular vessels associated with poor (limited/no response) outcomes.

In line with the hypothesis for psoriatic patients treated with NB-UVB previously proposed by Errichietti and Stinco [6], it is possible to speculate that the correlation between the therapeutic resistance of psoriatic plaques to calcipotriene plus betamethasone dipropionate aerosol foam and lesion localization on legs might be the result of a higher inflammatory activity related to a greater hydrostatic pressure in a subset of patients with microcirculation impairment/vessel dilation, with globular vessels potentially being a dermoscopic marker of higher hydrostatic pressure and, consequently, poor outcome. Accordingly, a higher hydrostatic pressure would lead to increased vessel permeability, with the consequent formation of a fibrin cuff around dermal capillaries that would cause tissue hypoxia, cell damage and the release of inflammatory mediators, which may in turn be responsible for treatment resistance [12-14]. However, it is also possible that the different pattern of vascularization (dotted vs. globular vessels) and the resulting response might be due to different cytokine milieus; to date, no specific evidence on this possibility is available in the literature, thus preventing us from further speculation in this regard. Future research is therefore needed to address this notion.

In the post-treatment analysis, we observed a significant tendency for relapse in the 4 weeks after a course of calcipotriol plus betamethasone dipropionate spray foam, with more than half of the lesions that had achieved improvement at the end of the therapy experiencing a loss of response. Importantly, relapse was strongly associated with the persistence of vascular structures on dermoscopy at the end of the therapy. This was observed in all of the lesions regardless the degree of improvement but also when only lesions reaching a complete/ nearly complete clinical healing at the end of the treatment were considered, with a risk of disease recurrence in the case of vessel persistence after the treatment being 17- and 21-fold higher, respectively.

A possible role of persistence of vascular changes in promoting disease relapse is supported by two well-known factors: (1) the onset of dermal microangiopathy in psoriasis usually precedes clinical relapse and (2) treatments favouring microangiopathy regression lead to a more stable clinical healing $[15,16]$. In such a scenario, it would be advisable to achieve a "dermoscopic healing", rather than a mere "clinical remission", to ensure a longer response retention. In our personal experience based on every other day assessment of two volunteers not included in this study and who displayed vessels persistence at the end of a standard 4-week treatment with calcipotriol plus betamethasone dipropionate spray foam despite clinical healing, we found that vessels disappearance occurred after 6 and 8 days, respectively, of "extra" application. However, specific systematic studies are needed to precisely understand how long the drug should be used to achieve dermoscopic vascular normalization.

\section{CONCLUSIONS}

The results of our study confirm that calcipotriol/betamethasone dipropionate spray foam is an effective treatment for plaque-type 
psoriasis, although clinical response can be adversely affected by local factors, including severe baseline infiltration and distribution of lesions on legs. We also found that the presence of globular vessels at baseline on dermoscopy is a negative response predictor as the presence of this type of vessel was significantly more frequent in non-responding lesions.

Interestingly, a significant tendency of the lesions to relapse in the 4 weeks after a standard course of calcipotriol plus betamethasone dipropionate spray foam was observed in this study. However, according to our findings, it would appear that disease recurrence is significantly associated to vessels persistence on dermoscopy at the end of the treatment, thereby suggesting that therapy should be continued until complete "dermoscopic healing" in order to optimize the treatment and achieve a longer remission.

\section{ACKNOWLEDGEMENTS}

Funding. This study was funded by LEO Pharma. No Rapid Service Fee was received by the journal for the publication of this article.

Authorship. All named authors meet the International Committee of Medical Journal Editors (ICMJE) criteria for authorship for this article, take responsibility for the integrity of the work as a whole, and have given their approval for this version to be published.

Disclosures. Enzo Errichetti is a member of the journal's Editorial Board. Margherita Croatto, Luca Arnoldo and Giuseppe Stinco have nothing to disclose.

Compliance with Ethics Guidelines. The study was conducted in accordance with the Helsinki Declaration of 1964 and its later amendments and all the participants gave their informed consent to take part in the study and use personal records/photos for scientific purpose. Local ethical committee approval was obtained from the Comitato Etico Unico
Regionale (C.E.U.R.) del Friuli Venezia Giulia (CEUR-2018-OS-140-ASUIUD).

Data Availability. The datasets generated during and/or analyzed during the current study are available from the corresponding author only on reasonable request.

Open Access. This article is licensed under a Creative Commons Attribution-NonCommercial 4.0 International License, which permits any non-commercial use, sharing, adaptation, distribution and reproduction in any medium or format, as long as you give appropriate credit to the original author(s) and the source, provide a link to the Creative Commons licence, and indicate if changes were made. The images or other third party material in this article are included in the article's Creative Commons licence, unless indicated otherwise in a credit line to the material. If material is not included in the article's Creative Commons licence and your intended use is not permitted by statutory regulation or exceeds the permitted use, you will need to obtain permission directly from the copyright holder. To view a copy of this licence, visit http://creativecommons.org/licenses/by$\mathrm{nc} / 4.0 /$.

\section{REFERENCES}

1. Gerdes S, Krakor M, Anger T, Hutt HJ, Körber A. Prospective, observational, non-interventional, multicentre study on the efficacy and tolerability of a new calcipotriol/betamethasone aerosol foam (Enstilar $\left.{ }^{\circledR}\right)$ in patients with plaque psoriasis under daily practice conditions. Dermatology. 2017;233: 425-34.

2. Pink AE, Jalili A, Berg P, et al. Rapid onset of action of calcipotriol/betamethasone dipropionate cutaneous foam in psoriasis, even in patients with more severe disease. J Eur Acad Dermatol Venereol. 2019;33:1116-23.

3. Jalili A, Lebwohl M, Stein Gold L, et al. Itch relief in patients with psoriasis: effectiveness of calcipotriol plus betamethasone dipropionate foam. J Eur Acad Dermatol Venereol. 2019;33:709-17.

4. Gallo L, Megna M, Cirillo T, et al. Psoriasis and skin pain: real-life effectiveness of calcipotriol plus 
betamethasone dipropionate in aerosol foam formulation. J Eur Acad Dermatol Venereol. 2019;33: 1312-5.

5. Stinco G, Trevisan G, Piccirillo F, et al. Pruritus in chronic plaque psoriasis: a questionnaire-based study of 230 Italian patients. Acta Dermatovenerol Croat. 2014;22:122-8.

6. Errichetti E, Stinco G. Clinical and dermoscopic response predictors in psoriatic patients undergoing narrowband ultraviolet B phototherapy: results from a prospective study. Int J Dermatol. 2018;57: 681-6.

7. Lallas A, Argenziano G, Zalaudek I, et al. Dermoscopic hemorrhagic dots: an early predictor of response of psoriasis to biologic agents. Dermatol Pract Concept. 2016;6:7-12.

8. Stinco G, Buligan C, Errichetti E, Valent F, Patrone P. Clinical and capillaroscopic modifications of the psoriatic plaque during therapy: observations with oral acitretin. Dermatol Res Pract. 2013;2013: 781942.

9. Errichetti E, Zalaudek I, Kittler H, et al. Standardization of dermoscopic terminology and basic dermoscopic parameters to evaluate in general dermatology (non-neoplastic dermatoses): an expert consensus on behalf of the International Dermoscopy Society. Br J Dermatol. 2020;182: 454-67.

10. Del Rosso JQ, Kircik LH. The effect of calcipotrienebetamethasone dipropionate aerosol foam versus vehicle on target lesions in moderate severity plaque psoriasis: focus on elbows and knees. J Drugs Dermatol. 2019;18:358-61.

11. Lind M, Nielsen KT, Schefe LH, et al. Supersaturation of calcipotriene and betamethasone dipropionate in a novel aerosol foam formulation for topical treatment of psoriasis provides enhanced bioavailability of the active ingredients. Dermatol Ther (Heidelb). 2016;6:413-25.

12. Pappas PJ, You R, Rameshwar P, et al. Dermal tissue fibrosis in patients with chronic venous insufficiency is associated with increased transforming growth factor-beta1 gene expression and protein production. J Vasc Surg. 1999;30:1129-45.

13. Cheatle TR, McMullin GM, Farrah J, et al. Skin damage in chronic venous insufficiency: does an oxygen diffusion barrier really exist? J R Soc Med. 1990;83:493-4.

14. Falanga V, Moosa HH, Nemeth AJ, et al. Dermal pericapillary fibrin in venous disease and venous ulceration. Arch Dermatol. 1987;123:620-3.

15. Campanati A, Goteri G, Simonetti O, et al. Angiogenesis in psoriatic skin and its modifications after administration of etanercept: videocapillaroscopic, histological and immunohistochemical evaluation. Int J Immunopathol Pharmacol. 2009;22:371-7.

16. Stinco G, Buligan C, Maione V, Valent F, Patrone P. Videocapillaroscopic findings in the microcirculation of the psoriatic plaque during etanercept therapy. Clin Exp Dermatol. 2013;38:633-7. 